\title{
The Admission Experience Survey Italian Version (I-AES): A factor analytic study on a sample of 156 acute psychiatric in-patients
}

\author{
Gabriele Mandarelli $^{\mathrm{a}, *}$, Giovanna Parmigiani ${ }^{\mathrm{a}}$, Federico Trobia ${ }^{\mathrm{b}}$, Gianmarco Tessari ${ }^{\mathrm{c}}$, \\ Paolo Roma ${ }^{\mathrm{a}}$, Massimo Biondi ${ }^{\mathrm{a}}$, Stefano Ferracuti ${ }^{\mathrm{a}}$ \\ a Department of Human Neurosciences, University of Rome "Sapienza", Italy \\ b Psychiatry Residency Training Program, Faculty of Medicine and Psychology, University of Rome "Sapienza", Italy \\ ${ }^{\mathrm{c}}$ Post-graduate School of Specialization in Neuropsychology, Psychology Department, University of Rome "Sapienza", Italy
}

\section{A R T I C L E I N F O}

\section{Keywords:}

Perceived coercion

Involuntary commitment

Severe mental illness

\begin{abstract}
A B S T R A C T
Coercive treatments are often regarded as an inevitable and yet highly debated feature of psychiatric care. Perceived coercion is often reported by patients involuntarily committed as well as their voluntary counterparts. The Admission Experience Survey (AES) is a reliable tool for measuring perceived coercion in mental hospital admission. We developed the Italian AES (I-AES) through translation back-translation and administered it to 156 acutely hospitalized patients (48\% women, $69 \%$ voluntarily committed) in two university hospitals in Rome (Policlinico Umberto I, Sant'Andrea Hospital). A principal component analysis (PCA) with equamax rotation was conducted. The I-AES showed good internal consistency (Cronbach's alpha $=0.90$ ); Guttmann split-half reliability coefficient was 0.90 . AES total score significantly differed between voluntary and involuntary committed patients $(5.08 \pm 4.1$ vs. $8.1 \pm 4.9, p<.05)$. PCA disclosed a three-factor solution explaining 59.3 of the variance. Some discrepancies were found between the factor structure of the I-AES and the original version. IAES total score was positively associated with numbers of previous involuntarily hospitalization $(r=0.20$, $\mathrm{p}<.05)$ and psychiatric symptoms' severity $(r=0.22, p<.02)$. I-AES and its proposed new factor structure proved to be reliable to assess perceived coercion in mental hospital admission. Consequently, it may represent a helpful instrument for the study and reduction of patients' levels of perceived coercion.
\end{abstract}

\section{Introduction}

Despite the significant efforts towards reducing the coercion associated with the treatment of severe mental disorders, compulsory treatment is often regarded to as an inevitable and yet highly debated feature of psychiatric care. Involuntary admission, forced pharmacological treatments, and physical restraint are common in psychiatric practice, but they pose serious ethical concerns due to their implications in terms of personal rights violations, and individuals' liberty limitations (Svindseth, Dahl, \& Hatling, 2007). Coercive practices vary through a continuum, ranging from subtle to overt coercion, and are often referred to as a last resort option, to be cautiously used only in such cases posing danger to self or others.

The subjective experience of being coerced when receiving medical care, the so-called perceived coercion, to be distinguished from objective coercion, has been reported also from those patients who have not been subjected to objective coercive measures (Bindman et al., 2005; Hiday, Swartz, Swanson, \& Wagner, 1997; Iversen, Høyer, Sexton, \& Grønli, 2002). Formal legal status has been reported to be variably associated with psychiatric patients' perceived coercion (Kjellin et al., 2004; Lidz et al., 1995). Commonly, coercion is associated with a negative subjective experience due to an intervention performed against the person's will, either using force or through the threat of using force, or which deviates from the patients' expectations. Specifically, feeling coerced in the mental health setting has been defined as perceiving that one does not have influence, control, freedom, or choice, or does not make the decision to enter the hospital (Gardner et al., 1993).

Perceived coercion in psychiatry proved to be influenced by sociodemographic and clinical variables, including age, marital status,

\footnotetext{
*Corresponding author at: Department of Human Neurosciences, University of Rome “Sapienza”, Policlinico Umberto I Hospital, Viale dell'Università 30 , 00185 Rome, IT, Italy.

E-mail addresses: gabriele.mandarelli@uniroma1.it (G. Mandarelli), giovanna.parmigiani@uniroma1.it (G. Parmigiani), federico.trobia@uniroma1.it (F. Trobia), gianmarco.tessari@uniroma1.it (G. Tessari), paolo.roma@uniroma1.it (P. Roma), massimo.biondi@uniroma1.it (M. Biondi), stefano.ferracuti@uniroma1.it (S. Ferracuti).
} 
gender, and ethnicity (Anestis et al., 2013; Bindman et al., 2005; Guarda et al., 2007; Hiday et al., 1997; Rain et al., 2003; Swartz \& Swanson, 2004). Female psychiatric patients tend to report higher coercion than their male counterparts (Fiorillo et al., 2012; Guarda et al., 2007; Hiday et al., 1997), and older patients tend to feel more coerced compared to younger ones (Guarda et al., 2007). The diagnosis of a psychotic disorder, substance abuse disorders, recent sexual abuse, poor insight, and low scores on measures of global functioning, have been linked with a higher level of perceived coercion (Bindman et al., 2005; Kjellin, Høyer, Engberg, Kaltiala-Heino, \& Sigurjónsdóttir, 2006; O'Donoghue et al., 2014). Individuals with hostile-dominant interpersonal styles report higher levels of perceived coercion (Anestis et al., 2013) and involuntarily admitted patients tend to perceive higher levels of coercion, when compared with voluntarily admitted ones (Lidz et al., 1995; Rain et al., 2003).

Among the tools used to measure psychiatric patients' perceived coercion, including the Cantril Ladder (Fiorillo et al., 2012; Iversen et al., 2002; Kjellin et al., 2004; Poulsen, 1999; Sorgaard, 2004), the Coercion Experience Scale (Bergk, Einsiedler, Flammer, \& Steinert, 2011; Borgeat \& Zullino, 2004; Guzmán-Parra et al., 2018; Mielau et al., 2016; Steinert, Birk, Flammer, \& Bergk, 2013), and the Nordic Admission Interview (Iversen et al., 2002; Iversen, Hoyer, \& Sexton, 2007; Kjellin et al., 2004), the most widely used is the Admission Experience Survey (AES) (Anestis et al., 2013; Bindman et al., 2005; Fiorillo et al., 2012; Fu, Chow, \& Lam, 2008; Gardner et al., 1993; Gowda, Noorthoorn, Kumar, Nanjegowda, \& Math, 2016; Guarda et al., 2007; Hiday et al., 1997; Iversen et al., 2002; Mielau et al., 2016; Poulsen, 1999; Seigel, Wallsten, Torsteinsdottir, \& Lindström, 2009).

The AES was developed by the MacArthur Research Network on Mental Health and the Law (Gardner et al., 1993), and it has been designed to assess psychiatric patients' perception of their hospitalization experience, including their perceived coercion. The AES explores the circumstances leading to hospital admission, patients' interaction with family members and clinical staff during hospital admission, and their perceptions of attempts by others to influence whether they entered the hospital (Gardner et al., 1993). The AES showed good psychometric characteristics and proved useful for measuring subjective coercion in different psychiatric clinical settings (Gardner et al., 1993). Researchers and clinicians from different countries chose the AES to measure patients' perception of coercion (Anestis et al., 2013; Bindman et al., 2005; Fiorillo et al., 2012; Fu et al., 2008; Gardner et al., 1993; Gowda et al., 2016; Hiday et al., 1997; Iversen et al., 2002; Iversen et al., 2007; Mielau et al., 2016; O'Donoghue et al., 2011; Poulsen, 1999; Seigel et al., 2009; Strack \& Schulenberg, 2009; Svindseth et al., 2007), and the scale has been translated into different languages ( $\mathrm{Fu}$, Chow, \& Lam, 2008; Gardner et al., 1993; Golay et al., 2017; Seigel, Wallsten, Torsteinsdottir, \& Lindström, 2009).

The subjective experience of being coerced may have several clinical implications. Perceived coercion has proved to be associated with poorer prognosis, a higher number of relapses and rehospitalizations, as well as lower adherence to treatment (Kaltiala-Heino, Laippala, \& Salokangas, 1997; Steinert et al., 2010). The subjective experience of coercion significantly decreases with the improvement in global functioning and the reduction of positive symptoms (Anestis et al., 2013; Fiorillo et al., 2012), and seems to not predict engagement with followup (Bindman et al., 2005; O'Donoghue et al., 2011). Nonetheless, other studies showed no clear association between clinical variables such as symptoms of psychoticism or depression and clear signs of hostility and perceived coercion (Kjellin et al., 2006; Poythress, Petrila, McGaha, \& Boothroyd, 2002).

Since perception of coercion associated with psychiatric treatment has been linked to the specific cultural and juridical framework, it might consequently vary among different countries and legislative systems providing different criteria for mental hospital admission. Moreover, the factors underlying perceived coercion, which are important when developing and defining preventive strategies to reduce perception of coercion, might also vary between different clinical populations. To our knowledge there are no standardized tools in Italian for assessing the subjective experience of coercion in psychiatric inpatients. The aim of the present study was to translate into Italian and validate the Admission Experience Survey (I-AES), to explore its psychometric properties including factorial structure. The availability of this instrument will allow the study of Italian psychiatric patients' perceived coercion during hospitalization. A second objective of the study was to investigate differences in perceived coercion in different diagnostic group of psychiatric patients as well as in voluntarily and involuntarily hospitalized patients.

\section{Materials and methods}

\subsection{Patients}

The present retrospective observational study included the evaluation of $n=200$ medical records of psychiatric patients who had been voluntarily or involuntarily hospitalized at two in-patient acute psychiatric units of the University Hospitals "Policlinico Umberto I" and "Sant'Andrea" of Rome.

Acute psychiatric admission to the hospital in Italy is preceded by an assessment at the emergency room. The patient can come to the hospital autonomously, also because of external pressures/decisions, usually from family members or the outpatient psychiatric services. Another possibility is that the patient arrives to the hospital through the ambulance, following an emergency situation. In this case he/she can arrive voluntarily or with a compulsory hospitalization provision already issued by the city mayor, upon request of two physicians.

We collected and analysed acute psychiatric patients' medical records between January 2015 and June 2015, which included the MiniMental State Examination (MMSE) (Folstein, Folstein, \& Mchugh, 1975) and the 24-item Brief Psychiatric Rating Scale (BPRS, version 4.0) (Ventura et al., 1993) as routinely collected data. Since there was a clinical need to measure the levels of coercion perceived by hospitalized psychiatric patients, we initially developed the I-AES and introduced it as part of the routine clinical evaluation. The AES was translated into Italian and back-translated by an independent postgraduate linguist, fluent in both English and Italian. The Italian version scale was then evaluated in focus groups followed by expert panel review.

Inclusion criteria were: minimum age of 18 years, fluent Italian; current MMSE score of 19 or higher. Patients were diagnosed according to the criteria of the Diagnostic and Statistical Manual of Mental Disorders- Fifth Edition, DSM-5 (American Psychiatric Association, 2013). All patients provided informed consent, allowing the use of anonymous clinical information to be used for research purposes. The study was carried out in accordance to the Declaration of Helsinki 1995 (as revised in Edinburgh 2000) and was approved by the Institutional Review Board.

\subsection{Admission experience survey}

The AES is a rapid assessment tool derived from a semi-structured interview originally proposed by Gardner and colleagues (Gardner et al., 1993). It consists of 16 items to be answered in a true-false format. The AES includes three subscales covering: a) "perceived coercion" (composed of 5 items focusing on influence, control, choice, freedom and idea, ranging from 0 to 5 , where a score of 5 reflects the maximum degree of subjective coercion); b) "negative pressures scale" (composed of six items, evaluating if in the process of hospitalization threats and force were applied, ranging from 0 to 6); c) "voice scale" (composed of 3 items assessing to what extent patients experienced that they had a chance to express their opinions, and having others take into account their viewpoints, ranging from 0 to 3). Item 9 (No one seemed to want to know whether I wanted to come into the hospital) of the AES was eventually dropped from these scales. Item 16 explores the affective 
reactions to hospitalization (How did being admitted to the hospital make you feel? Did it make you feel: a) Angry; b) Sad; c) Pleased; d) Relieved; e) Confused; f) Frightened). (see The MacArthur Coercion Study, 2001).

\subsection{Clinical and cognitive assessment}

The 24-item BPRS, version 4.0 evaluated current psychiatric symptoms severity (Ventura et al., 1993). The scale is composed of 24 items investigating the main psychiatric signs and symptoms. Each item is rated on a 7-point Likert scale ranging from 1 (not present) to 7 (extremely severe). Four BPRS factor scores were calculated, i.e. manic excitement/disorganization, anxiety/depression, negative symptoms, and positive symptoms (Ruggeri et al., 2005).

Cognitive functioning was assessed with the MMSE (Folstein et al., 1975). The MMSE is an 11-items scale screening tool and was developed to be a practical method for grading the cognitive state of patients. The total score results from the sum of each item, ranging from a minimum of 0 , indicating maximum cognitive deficit, to 30 , which indicates good cognitive performance.

\section{Statistics}

Statistical analyses were performed with the Statistical Software for Social Sciences v. 17.0 (SPSS). The alpha value was set to 0.05 , all tests were two-tailed. Differences between groups in continuous variables were analysed by the independent sample $t$-test. The chi-square test or Fisher's exact-test was used for comparisons between categorical variables. Correlations were calculated by the Pearson's correlation coefficient. We carried out an exploratory factor analysis by principal component analysis on the I-AES items through Eigenvalue method $>1$ in order to extract the factors, also observing the scree plot. We rotated the factors by an oblique rotation (equamax), and we considered only the items with load factor $>0.5$. We analysed the I-AES internal consistency through Cronbach's alpha. A subsample of $N=26$ patients, who were re-administered the I-AES within 10-14 days, was used to assess test-retest reliability by paired sample $t$-test confronting patients' I-AES total scores.

\section{Results}

From the $N=200$ medical records originally screened 156 were included. The study sample of acutely admitted psychiatric patients comprised 49 involuntarily hospitalized patients (31.4\%). Seventy-one percent of patients suffered from schizophrenia spectrum disorder or bipolar disorder and had a history of previous psychiatric hospitalization (Table 1). The I- AES total score ranged from 0 to 15, with a mean score of $5.9(\mathrm{SD}=4.5)$. Mean MMSE total score indicated no evidence of cognitive dysfunction in the sample overall (25.6; $\mathrm{SD}=2.7$ ).

Principal Component Analysis (PCA) disclosed a 3-factor solution (Table 2) explaining $59.3 \%$ of the total variance. The first I-AES factor, which we named "perceived coercion" comprised 7 items referring to the subjective experience of coercion. These 7 items included the 5 items originally designed by Gardner and colleagues (Gardner et al., 1993) in the "perceived coercion subscale", referring to influence (I had more influence than anyone else on whether I came into the hospital), control (I had a lot of control over whether I went into the hospital), "choice" (I chose to come into the hospital), freedom (I felt free to do what I wanted about coming into the hospital), idea (It was my idea to come into the hospital) plus one item from Gardner's "negative pressure scale" (They said they would make me come into the hospital) and one item from Gardner's "voice scale" (My opinion about coming into the hospital didn't matter).

The second I-AES factor, named "external pressure", comprised 5 items referring to the external pressure experienced and included 5 of 6 items from Gardner and colleagues' "negative pressure scale". The third IAES factor, named "choice expression" included three items referring to the patients' perception of the possibility of expressing his/her opinion and included 2 of 3 items from Gardner's "voice scale", and item 9 (No one seemed to want to know whether I wanted to come into the hospital) which was eventually dropped from the original Gardner's scale.

Independent sample $t$-test disclosed no differences in the BPRS total scores nor in MMSE scores between voluntarily and involuntarily hospitalized patients (Table 3). Higher levels of perceived coercion emerged in involuntary patients than in voluntary ones, as measured by the I-AES total score and the 3 subscale scores ("perceived coercion", "external pressure" and "choice expression") (Table 3).

Total I-AES total score was positively associated with numbers of previous involuntarily hospitalizations $(r=0.20, p<.05)$ and psychiatric symptoms' severity $(r=0.22, p<.02)$ as measured by the BPRS total score. Manic excitement/disorganization symptoms were positively associated with "perceived coercion" factor $(r=0.21$, $p<.05)$, "external pressure" factor $(r=0.32, p<.01)$, and "choice expression" factor $(r=0.19, p<.05)$. Anxiety and depressive symptoms were negatively associated with "perceived coercion" factor $(r=-0.2, p<.02)$, and "external pressure" factor $(r=-0.23$, $p<.01)$. Negative symptoms were positively associated with "perceived coercion" factor $(\mathrm{r}=0.19, p<.04)$, while positive symptoms were positively associated with "external pressure" factor $(r=0.20$, $p<.03)$.

The I-AES showed good internal consistency (Cronbach's alpha $=0.90$ ). Guttmann split-half reliability coefficient was 0.90 . Paired sample $t$-test disclosed no significant differences between test and retest I-AES total scores, paired sample correlation was 0.62 .

Table 1

Socio-demographic characteristics of the acutely hospitalized psychiatric patients.

\begin{tabular}{|c|c|c|c|c|}
\hline & Total sample $(N=156)$ & $\begin{array}{l}\text { Involuntarily hospitalized patients } \\
(N=49)\end{array}$ & $\begin{array}{l}\text { Voluntarily hospitalized patients } \\
(N=107)\end{array}$ & $p$ \\
\hline Age, years, M (SD) & $40.5(12.7)$ & $39.6(10.3)$ & $40.9(13.7)$ & $\mathrm{Ns}^{*}$ \\
\hline Women, n (\%) & $74(47)$ & $27(55.1)$ & $47(43.9)$ & Ns \\
\hline Disease duration, years, $\mathrm{M}(\mathrm{SD})$ & $12.1(9.4)$ & $12.5(8.4)$ & $11.9(9.8)$ & $\mathrm{Ns}^{*}$ \\
\hline \multicolumn{5}{|l|}{ Diagnosis, n (\%) } \\
\hline Schizophrenia spectrum disorders & $56(35.9)$ & $19(38.8)$ & 37 (34.6) & Ns \\
\hline $\begin{array}{l}\text { Number of previous voluntary psychiatric hospitalizations, M } \\
\text { (SD) }\end{array}$ & $1.4(2.9)$ & $1.9(4.5)$ & $1.1(1.7)$ & $\mathrm{Ns}^{*}$ \\
\hline $\begin{array}{l}\text { Number of previous involuntary psychiatric hospitalizations, } \\
\text { M (SD) }\end{array}$ & $0.7(2.6)$ & $1.5(4.4)$ & $0.4(0.8)$ & $\mathrm{Ns}^{*}$ \\
\hline
\end{tabular}

Note: $p$ values by Chi-square test; * $p$ values by independent sample t-test; $\mathrm{Ns}=$ not-significant. 
Table 2

I-AES Principal component analysis.

\begin{tabular}{|c|c|c|c|}
\hline Admission Experience Survey items & $\begin{array}{l}\text { Perceived Coercion } \\
\text { (factor } 1 \text { ) }\end{array}$ & $\begin{array}{l}\text { External pressure } \\
\text { (factor 2) }\end{array}$ & $\begin{array}{l}\text { Choice expression } \\
\text { (factor } 3 \text { ) }\end{array}$ \\
\hline 7. It was my idea to come into the hospital & 0.78 & & \\
\hline 15. I had more influence than anyone else on whether I came into the hospital & 0.73 & & \\
\hline 4. I chose to come into the hospital & 0.69 & & \\
\hline 1. I felt free to do what I wanted about coming into the hospital & 0.63 & & \\
\hline 13. My opinion about coming into the hospital didn't matter & 0.60 & & \\
\hline 14. I had a lot of control over whether I went into the hospital & 0.59 & & \\
\hline 11. They said they would make me come into the hospital & 0.50 & & \\
\hline 10. I was threatened with commitment & & 0.86 & \\
\hline 6. Someone threatened me to get me come into the hospital & & 0.79 & \\
\hline 8. Someone physically tried to make me come into the hospital & & 0.63 & \\
\hline 12. No one tried to force me to come into the hospital & & 0.53 & \\
\hline 2. People tried to force me to come into the hospital & & 0.51 & \\
\hline 5. I got to say what I wanted about coming into the hospital & & & 0.75 \\
\hline 3. I had enough of a chance to say whether I wanted to come into the hospital & & & 0.67 \\
\hline 9. No one seemed to want to know whether I wanted to come into the hospital & & & 0.60 \\
\hline Cronbach's alpha & 0.84 & 0.79 & 0.71 \\
\hline Variance explained & 25.1 & 19.1 & 15.1 \\
\hline
\end{tabular}

\section{Discussion}

Results from this study suggest that the I-AES, administered to a sample of acute psychiatric in-patients, has satisfactory psychometric characteristics. To the best of our knowledge, there is just one other study evaluating Italian psychiatric patients' subjective experience of coercion during hospital treatment (Fiorillo et al., 2012). Our results extend the existing data on the subjective experience of coercion in psychiatric hospitalization (Anestis et al., 2013; Bindman et al., 2005; Fiorillo et al., 2012; Fu et al., 2008; Gardner et al., 1993; Gowda et al., 2016; Guarda et al., 2007; Hiday et al., 1997; Iversen et al., 2002; Mielau et al., 2016; O'Brien \& Golding, 2003; Poulsen, 1999; Seigel et al., 2009; Sorgaard, 2004) and provide an empirical factorial structure of the AES.

Involuntary hospitalized patients included in our sample presented higher perceived coercion during hospitalization compared to voluntarily ones, as measured by I-AES total score and three subscales. A possible confounding role played by symptoms severity or different cognitive functioning can be excluded because we found no differences in such variables between voluntarily and involuntarily hospitalized acute psychiatric patients. In interpreting this result we should consider that the criteria for involuntary psychiatric hospitalization in Italy rely on the presence of a mental disorder and need for treatment (as in Spain and Sweden) and not, as in most other countries, on patients' dangerousness to themselves or others (Dressing \& Salize, 2004). This may imply that the involuntariness of the hospitalization is the main factor in determining the experience of being coerced.

In the whole sample, perceived coercion was associated with the number of previous involuntary psychiatric hospitalizations, while no association emerged with number of previous psychiatric hospitalizations. This might underly that patients with a history of a higher number of previous involuntary hospitalizations tend to show a more severe psychiatric symptomatology, a poor adherence to treatment, and more aversity towards psychiatric care, thus probably resulting in the experience of feeling more coerced during subsequent hospital admissions.

Psychiatric symptoms severity was associated with the experience of being coerced. Those patients with higher manic excitement/disorganization symptoms tended to feel more coerced, to report more threats and force application during the process of hospitalization, and a lower chance to have a say about the admission or having others consider their viewpoints. The severity of psychiatric symptoms can be associated with a lack of insight and the need of a therapeutic approach which can imply the use of subtle or overt coercive measures. However, the absence of empirical data about objective coercion limits the possibility to verify this hypothesis in the present study. In other studies by our group the presence of mania was associated with involuntary hospitalization and a poorer capacity to consent to treatment (Carabellese et al., 2017; Mandarelli et al., 2017; Mandarelli et al., 2014). It would be interesting to investigate the presence of an association between perceived coercion and capacity to consent to treatment in clinical populations and how cognitive and psychiatric symptoms play a role in this picture. Anxiety and depressive symptoms were inversely associated with perceived coercion and external/negative pressure; this may be due to the fact that patients affected by depression are more inclined to voluntarily or passively adhere to psychiatric treatment and hospital admission. Finally, negative symptoms were associated with a higher perception of coercion, while positive symptoms were associated

Table 3

Clinical characteristics of the acutely hospitalized psychiatric patients.

\begin{tabular}{|c|c|c|c|c|}
\hline & Total sample $(\mathrm{N}=156)$ & $\begin{array}{l}\text { Involuntarily hospitalized patients } \\
(\mathrm{N}=49)\end{array}$ & $\begin{array}{l}\text { Voluntarily hospitalized patients } \\
(\mathrm{N}=107)\end{array}$ & $p$ \\
\hline MMSE total score, M (SD) & $25.6(2.7)$ & $26.5(2.6)$ & $25.8(2.7)$ & Ns \\
\hline BPRS anxiety/depression score, M (SD) & $16.1(6.4)$ & $14.5(5.7)$ & $16.8(6.7)$ & Ns \\
\hline BPRS manic excitement/disorganization, M (SD) & $12.7(5.5)$ & $14.3(5.3)$ & $12.1(5.6)$ & $<0.05$ \\
\hline BPRS negative symptoms score, M (SD) & $12.6(4.5)$ & $12.6(4.2)$ & $12.6(4.7)$ & Ns \\
\hline BPRS positive symptoms score, M (SD) & $12.6(6.2)$ & $14.8(5.6)$ & $11.7(6.3)$ & $<0.01$ \\
\hline BPRS total score, M (SD) & $54.2(13.0)$ & $57.1(11.3)$ & $53.1(13.5)$ & Ns \\
\hline I-AES, total score, M (SD) & $5.9(4.5)$ & $8.1(4.9)$ & $5.1(4.1)$ & $<0.001$ \\
\hline I-AES perceived coercion, $M$ (SD) & $3.4(2.5)$ & $4.4(2.5)$ & $3.0(2.3)$ & $<0.001$ \\
\hline I-AES external pressure, M (SD) & $1.6(1.7)$ & $2.2(1.9)$ & $1.2(1.5)$ & $<0.001$ \\
\hline I-AES choice expression, M (SD) & $1.0(1.1)$ & $1.3(1.1)$ & $0.8(1.0)$ & $<0.05$ \\
\hline
\end{tabular}

Note: $p$ values by independent sample $t$-test. Ns $=$ not-significant. 
with the experience of external/negative pressure. This last result may be explained by patients' distorted perception, including that of their disease, which might lead to consider hospitalization as an inappropriate.

Exploratory factor analysis disclosed some differences between the construct of the I-AES and the original AES. One item from Gardner's "negative pressure scale" (They said they would make me come into the hospital) and one item from Gardner's "voice scale" (My opinion about coming into the hospital didn't matter) loaded on the "perceived coercion" IAES subscale. Item 9 (No one seemed to want to know whether I wanted to come into the hospital), which did not load on Gardner's subscales, in the present study, loaded on the "choice expression factor". This result may be due to socio-cultural variables that led Italian psychiatric patients' population to perceive these two items with a slightly different meaning compared to the ones of the original English version. Another possible explanation relies on the different methods used by the authors to validate the scale, which not included a factor analysis.

Three other studies performing a factor analysis of the AES disclosed differences from the original subscales (Fu et al., 2008; Golay et al., 2017; Seigel et al., 2009). These discrepancies might underline the role of cultural components on psychiatric patients' perceived coercion and deserve further investigation on larger and heterogenous samples. Possible limitations of this study that could restrict the generalizability of our results, are the relatively small sample size, the possible presence of recall bias and the absence of the collection of episodes of actual use of force, such as physical restraint or forcible medication. Despite these caveats, the I-AES has proved to be a reliable tool that could be helpful to investigate the perception of coercion among psychiatric patients, in order to improve the quality of care project.

\section{Conclusions}

The Italian version of AES proved to be a psychometrically solid assessment of "perceived coercion", "external pressure" and "choice expression" related to psychiatric hospital admission. The use of this brief tool can be useful in assessing psychiatric in-patients' experience in order to identify and reduce the factors that contribute to the perception of coercion and develop treatments focused on patients need.

\section{Competing interests}

The authors have declared that no competing interests exist.

\section{Funding}

This research did not receive any specific grant from funding agencies in the public, commercial, or not-for-profit sectors.

\section{References}

American Psychiatric Association (2013). Diagnostic and statistical manual of mental disorders (fifth edition). Washington, DC: American Psychiatric Association.

Anestis, A., Daffern, M., Thomas, S. D. M., Podubinski, T., Hollander, Y., Lee, S., \& Kulkarni, J. (2013). Predictors of perceived coercion in patients admitted for psychiatric hospitalization and the stability of these perceptions over time. Psychiatry, Psychology and Law, 20(4), 492-503. https://doi.org/10.1080/13218719.2012. 712833.

Bergk, J., Einsiedler, B., Flammer, E., \& Steinert, T. (2011). A randomized controlled comparison of seclusion and mechanical restraint in inpatient settings. Psychiatric Services, 62(11), 1310-1317.

Bindman, J., Reid, Y., Szmukler, G., Tiller, J., Thornicroft, G., \& Leese, M. (2005). Perceived coercion at admission to psychiatric hospital and engagement with followup-a cohort study. Social Psychiatry and Psychiatric Epidemiology, 40(2), 160-166. https://doi.org/10.1007/s00127-005-0861-x.

Borgeat, F., \& Zullino, D. (2004). Attitudes concerning involuntary treatment of mania: Results of a survey within self-help organizations. European Psychiatry, 19(3), 155-158.

Carabellese, F., Mandarelli, G., La Tegola, D., Parmigiani, G., Ferracuti, S., Quartesan, R., \& Catanesi, R. (2017). Mental capacity and capacity to consent: Multicentric study in a involuntary psychiatric hospitalized patients sample. Rivista di Psichiatria, 52(2),
67-74. https://doi.org/10.1708/2679.27442.

Dressing, H., \& Salize, H. J. (2004). Compulsory admission of mentally ill patients in European Union Member States. Social Psychiatry and Psychiatric Epidemiology, 39, 797-803.

Fiorillo, A., Giacco, D., De Rosa, C., Kallert, T., Katsakou, C., Onchev, G., \& Priebe, S. (2012). Patient characteristics and symptoms associated with perceived coercion during hospital treatment. Acta Psychiatrica Scandinavica, 125(6), 460-467. https:// doi.org/10.1111/j.1600-0447.2011.01809.x.

Folstein, M., Folstein, S., \& Mchugh, P. (1975). Mini-mental state: A practical guide for grading the cognitive state of the patient for the physician. Journal of Psychiatric Research, 12(3), 189-198.

Fu, J. C., Chow, P. P., \& Lam, L. C. (2008). The experience of admission to psychiatric hospital among Chinese adult patients in Hong Kong. BMC Psychiatry, 8, 86. https:// doi.org/10.1186/1471-244X-8-86.

Gardner, W., Hoge, S. K., Bennett, N., Roth, L. H., Lidz, C. W., Morrahan, J., \& Mulvey, E. P. (1993). Two Scales for measuring Patients' Perceptions for Coercion during Mental H ospitai Admission. Behavioral Sciences and the Law, 11, 307-321.

Golay, P., Semlali, I., Beuchat, H., Pomini, V., Silva, B., Loutrel, L., \& Bonsack, C. (2017). Perceived coercion in psychiatric hospital admission: Validation of the French-language version of the MacArthur Admission experience survey. BMC Psychiatry, 17(1), 357. https://doi.org/10.1186/s12888-017-1519-4.

Gowda, G. S., Noorthoorn, E. O., Kumar, C. N., Nanjegowda, R. B., \& Math, S. B. (2016). Clinical correlates and predictors of perceived coercion among psychiatric inpatients: A prospective pilot study. Asian Journal of Psychiatry, 22, 34-40. https://doi.org/10. 1016/j.ajp.2016.04.004.

Guarda, A. S., Pinto, A. M., Coughlin, J. W., Hussain, S., Haug, N. A., \& Heinberg, L. J. (2007). Perceived coercion and change in perceived need for admission in patients hospitalized for eating disorders. The American Journal of Psychiatry, 164, 108-114.

Guzmán-Parra, J., Aguilera-Serrano, C., García-Sanchez, J. A., García-Spínola, E., TorresCampos, D., Villagrán, J. M., \& Mayoral-Cleries, F. (2018). Experience coercion, posttraumatic stress, and satisfaction with treatment associated with different coercive measures during psychiatric hospitalization. International Journal of Mental Health Nursing. https://doi.org/10.1111/inm.12546 (Epub ahead of print).

Hiday, V. A., Swartz, M. S., Swanson, J., \& Wagner, H. R. (1997). Patient perceptions of coercion in mental hospital admission. International Journal of Law and Psychiatry, 20(2), 227-241.

Iversen, K. I., Høyer, G., Sexton, H., \& Grønli, O. K. (2002). Perceived coercion among patients admitted to acute wards in Norway. Nordic Journal of Psychiatry, 56(6), 433-439.

Iversen, K. I., Hoyer, G., \& Sexton, H. C. (2007). Coercion and patient satisfaction on psychiatric acute wards. International Journal of Law and Psychiatry, 30(6), 504-511. https://doi.org/10.1016/j.ijlp.2007.09.001.

Kaltiala-Heino, R., Laippala, P., \& Salokangas, R. K. R. (1997). Impact of coercion on treatment outcome. International Journal of Law and Psychiatry, 20(3), 311-322.

Kjellin, L., Andersson, K., Bartholdson, E., Candefjord, I. L., Holmstrom, H., Jacobsson, L., \& Ostman, M. (2004). Coercion in psychiatric care - patients' and relatives' experiences from four Swedish psychiatric services. Nordic Journal of Psychiatry, 58(2), 153-159. https://doi.org/10.1080/08039480410005549.

Kjellin, L., Høyer, G., Engberg, M., Kaltiala-Heino, R., \& Sigurjónsdóttir, M. (2006). Differences in perceived coercion at admission to psychiatric hospitals in the Nordic countries. Social Psychiatry and Psychiatric Epidemiology, 41(3), 241-247.

Lidz, C. W., Hoge, S. K., Gardner, W., Bennett, N. S., Monahan, J., Mulvey, E. P., \& Roth, L. H. (1995). Perceived coercion in mental hospital admission. Pressures and process. Archives of General Psychiatry, 52(12), 1034-1039.

Mandarelli, G., Carabellese, F., Parmigiani, G., Bernardini, F., Pauselli, L., Quartesan, R., \& Ferracuti, S. (2017). Treatment decision-making capacity in non-consensual psychiatric treatment: A multicentre study. Epidemiology and Psychiatric Sciences, 1-8. https://doi.org/10.1017/S2045796017000063.

Mandarelli, G., Tarsitani, L., Parmigiani, G., Polselli, G. M., Frati, P., Biondi, M., \& Ferracuti, S. (2014). Mental capacity in patients involuntarily or voluntarily receiving psychiatric treatment for an acute mental disorder. Journal of Forensic Sciences, 59(4), 1002-1007. https://doi.org/10.1111/1556-4029.12420.

Mielau, J., Altunbay, J., Gallinat, J., Heinz, A., Bermpohl, F., Lehmann, A., \& Montag, C. (2016). Subjective experience of coercion in psychiatric care: A study comparing the attitudes of patients and healthy volunteers towards coercive methods and their justification. European Archives of Psychiatry and Clinical Neuroscience, 266(4), 337-347. https://doi.org/10.1007/s00406-015-0598-9.

O'Brien, A. J., \& Golding, C. G. (2003). Coercion in mental healthcare: The principle of least coercive care. Journal of Psychiatric and Mental Health Nursing, 10, 167-173.

O'Donoghue, B., Lyne, J., Hill, M., O'Rourke, L., Daly, S., Larkin, C., \& O'Callaghan, E. (2011). Perceptions of involuntary admission and risk of subsequent readmission at one-year follow-up: The influence of insight and recovery style. Journal of Mental Health, 20(3), 249-259. https://doi.org/10.3109/09638237.2011.562263.

O'Donoghue, B., Roche, E., Shannon, S., Lyne, J., Madigan, K., \& Feeney, L. (2014). Perceived coercion in voluntary hospital admission. Psychiatry Research, 215(1), 120-126. https://doi.org/10.1016/j.psychres.2013.10.016.

Poulsen, H. D. (1999). Perceived coercion among committed, detained, and voluntary patients. International Journal of Law and Psychiatry, 22(2), 167-175.

Poythress, N. G., Petrila, J., McGaha, A., \& Boothroyd, R. (2002). Perceived coercion and procedural justice in the Broward mental health court. International Journal of Law and Psychiatry, 25, 517-533.

Rain, S. D., Williams, V. F., Robbins, P. C., Monahan, J., Steadman, H. J., \& Vesselinov, R. (2003). Perceived coercion at hospital admission and adherence to mental health treatment after discharge. Psychiatric Services, 54, 103-105.

Ruggeri, M., Koeter, M., Schene, A., Bonetto, C., Vazquez-Barquero, J. L., Becker, T., \& Group, E. S (2005). Factor solution of the BPRS-expanded version in schizophrenic 
outpatients living in five European countries. Schizophrenia Research, 75(1), 107-117. https://doi.org/10.1016/j.schres.2004.05.017.

Seigel, K., Wallsten, T., Torsteinsdottir, G., \& Lindström, E. (2009). Perception of coercion: A pilot study using the Swedish version of the Admission experience Scale. Nordic Journal of Psychiatry, 51(1), 49-54. https://doi.org/10.3109/ 08039489709109084.

Sorgaard, K. W. (2004). Patients' perception of coercion in acute psychiatric wards. An intervention study. Nordic Journal of Psychiatry, 58(4), 299-304. https://doi.org/10. 1080/08039480410005819.

Steinert, T., Birk, M., Flammer, E., \& Bergk, J. (2013). Subjective distress after seclusion or mechanical restraint: 1-year follow-up of a randomized controlled study. Psychiatric Services, 64(10), 1012-1017. https://doi.org/10.1176/appi.ps. 201200315.

Steinert, T., Lepping, P., Bernhardsgrütter, R., Conca, A., Hatling, T., Janssen, W., \& Whittington, R. (2010). Incidence of seclusion and restraint in psychiatric hospitals: A literature review and survey of international trends. Soc. Psychiatry Psychiat Epidemiol, 45(9), 889-897. https://doi.org/10.1007/s00127-009-0132-3.

Strack, K. M., \& Schulenberg, S. E. (2009). Understanding empowerment, meaning, and perceived coercion in individuals with serious mental illness. Journal of Clinical Psychology, 65(10), 1137-1148. https://doi.org/10.1002/jclp.20607.

Svindseth, M. F., Dahl, A. A., \& Hatling, T. (2007). Patients' experience of humiliation in the admission process to acute psychiatric wards. Nordic Journal of Psychiatry, 61(1), 47-53. https://doi.org/10.1080/08039480601129382.

Swartz, M. S., \& Swanson, J. W. (2004). Involuntary outpatient commitment, community treatment orders, and assisted outpatient treatment: what's in the data? Canadian Journal of Psychiatry, 49(9), 585-591.

The MacArthur Coercion Study - Admission Experience Survey: Short Form (2001) http://www.macarthur.virginia.edu/shortform.html, Accessed date: 9 May 2018.

Ventura, J., Lukoff, D., Nuechterlein, K. H., Liberman, R. P., Green, M., \& Shaner, A. (1993). Appendix 1: Brief Psychiatric Rating Scale (BPRS) Expanded Version (4.0) scales, anchor points and administration manual. International Journal of Methods in Psychiatric Research, 3, 227-243.

World Medical Association. Declaration of Helsinki: Ethical principles for medical research involving human subjects, 52nd WMA General Assembly, Edinburgh, Scotland, October 2000. 